\title{
Large cross-free sets in Steiner triple systems
}

\author{
András Gyárfás * \\ Alfréd Rényi Institute of Mathematics \\ Hungarian Academy of Sciences \\ Budapest, P.O. Box 127 \\ Budapest, Hungary, H-1364 gyarfas .andras@renyi.mta.hu
}

September 21, 2015

\begin{abstract}
A cross-free set of size $m$ in a Steiner triple system $(V, \mathcal{B})$ is three pairwise disjoint $m$-element subsets $X_{1}, X_{2}, X_{3} \subset V$ such that no $B \in \mathcal{B}$ intersects all the three $X_{i}$-s. We conjecture that for every admissible $n$ there is an $\operatorname{STS}(n)$ with a cross-free set of size $\left\lfloor\frac{n-3}{3}\right\rfloor$ which if true, is best possible. We prove this conjecture for the case $n=18 k+3$, constructing an STS $(18 k+3)$ containing a cross-free set of size $6 k$. We note that some of the 3-bichromatic STSs, constructed by Colbourn, Dinitz and Rosa, have cross-free sets of size close to $6 k$ (but cannot have size exactly $6 k$ ).

The constructed STS $(18 k+3)$ shows that equality is possible for $n=18 k+3$ in the following result: in every 3-coloring of the blocks of any Steiner triple system $\operatorname{STS}(n)$ there is a monochromatic connected component of size at least $\left\lceil\frac{2 n}{3}\right\rceil+1$ (we conjecture that equality holds for every admissible $n$ ).

The analogue problem can be asked for $r$-colorings as well, if $r-1 \equiv$ $1,3(\bmod 6)$ and $r-1$ is a prime power, we show that the answer is the same as in case of complete graphs: in every $r$-coloring of the blocks of any $\operatorname{STS}(n)$, there is a monochromatic connected component with at least $\frac{n}{r-1}$ points, and this is sharp for infinitely many $n$.
\end{abstract}

\section{Introduction}

A hyperwalk in a hypergraph $H=(V, E)$ is a sequence $v_{1}, e_{1}, v_{2}, e_{2}, \ldots, v_{t-1}, e_{t-1}, v_{t}$ of vertices and edges such that for all $1 \leq i<t$ we have $v_{i} \in e_{i}$ and $v_{i+1} \in e_{i}$.

*Supported in part by OTKA K104373. 
We say that $v \sim w$, if there is a hyperwalk from $v$ to $w$. The relation $\sim$ is an equivalence relation, and the subhypergraphs induced by its classes are called the connected components of $H$. A vertex $v$ that is not covered by any edge forms a trivial component with one vertex $v$ and no edge.

The size of the largest monochromatic component in edge colorings of complete graphs and hypergraphs is well investigated, for a present survey see [5]. For example, in every 3-coloring of the edges of the complete graph $K_{n}$ there is a monochromatic connected component of size at least $n / 2$ and in every 3-coloring of the edges of $K_{n}^{3}$, the complete 3-uniform hypergraph, there is a monochromatic spanning component. What happens in between, when the blocks of a Steiner triple system $(V, \mathcal{B})$ are colored? For example, in every coloring of the blocks of STS(9) with 3 colors, there is a monochromatic connected component of size at least 7 but in the 4-coloring of its blocks defined by the four parallel classes, every component in every color has only 3 points. Let $f(n)$ denote the largest $m$ such that in every 3-coloring of the blocks of any $\operatorname{STS}(n)$ there is a monochromatic connected component on at least $m$ points. It is easy to see that $f(7)=6, f(9)=7$. Our main result is the following.

Theorem 1. $f(6 k+3) \geq 4 k+3$ with equality if $k$ is divisible by 3. Moreover, $f(6 k+1) \geq 4 k+2$.

In fact, the inequalities of Theorem 11 are probably always sharp (one can easily check the cases $k=1,2)$ :

Conjecture 2. For every positive integer $k, f(6 k+1)=4 k+2, f(6 k+3)=4 k+3$.

Three pairwise disjoint $m$-element sets of points, $X_{1}, X_{2}, X_{3} \subset V$, is a cross-free set of size $m$ in a Steiner triple system $(V, \mathcal{B})$ if no block $B \in \mathcal{B}$ intersects each $X_{i}$ in exactly one point. To obtain the upper bound in Theorem 1, we need some STS with a cross-free set of size almost $n / 3$. In Theorem 4 we construct an $\operatorname{STS}(6 k+3)$ for the case $k \equiv 0(\bmod 3)$ which contains a cross-free set of size $2 k$ (and this is best possible).

It is worth noting that constructions of Colbourn, Dinitz and Rosa in [2] provides $\operatorname{STS}(n)$-s with cross-free sets of size asymptotic to $n / 3$. They construct 3 -bichromatic STSs where all points are partitioned into $X_{1}, X_{2}, X_{3}$ so that every block intersects precisely two of the $X_{i}$-s and they can also control the sizes of the $X_{i}$ s. In particular, they provide 3-bichromatic STS(n)s where the sizes are nearly equal to $n / 3$. However, it follows easily that in 3-bichromatic $\operatorname{STS}(n)$ s with $\left|X_{1}\right| \leq\left|X_{2}\right| \leq\left|X_{3}\right|, n / 3-\left|X_{i}\right|$ tends to infinity with $n$. Therefore, to achieve a cross-free set of size $2 k$ in an STS $(6 k+$ 3 ) the number of blocks inside the $X_{i}$ s tends to infinity with $n$.

To see the connection of cross-free sets to $f(n)$, let $G(n)$ be the size of the largest cross-free set present in some $\operatorname{STS}(n)$. 
Lemma 3. $f(n) \leq n-G(n)$.

Proof. Suppose $\left|X_{1}\right|=\left|X_{2}\right|=\left|X_{3}\right|=G(n)$ for a cross-free set $X_{1}, X_{2}, X_{3} \subset V$ in some $\operatorname{STS}(n)$. Then coloring any block $B$ with the smallest $i$ such that $B \subset V \backslash X_{i}$, we have a 3-coloring of the blocks with one nontrivial monochromatic connected component of size $n-G(n)$ in each color.

The next result implies the equality $f(6 k+3)=4 k+3$ for $k$ divisible by 3 in Theorem 1 .

Theorem 4. For $n=18 k+3, G(n)=6 k$.

In fact, Theorem 4 probably can be extended, it would imply Conjecture 2

Conjecture 5. $G(6 k+3)=2 k, G(6 k+1)=2 k-1$.

It is easy to see that Conjecture 5 is sharp (if true). Indeed, a cross-free set of size $2 k+1$ in an $\operatorname{STS}(6 k+3)$ would mean that there are at most $3\left(\begin{array}{c}2 k+1 \\ 2\end{array}\right)$ blocks and that is less than $\left(\begin{array}{c}6 k+3 \\ 2\end{array}\right) / 3$. Similarly, a cross-free set of size $2 k$ in an $\operatorname{STS}(6 k+1)$ would show that there are at most $3 k+3\left(\begin{array}{c}2 k \\ 2\end{array}\right)$ blocks, less than $\left(\begin{array}{c}6 k+1 \\ 2\end{array}\right) / 3$.

One can define $f_{r}(n)$ similarly for $r$-colorings of blocks. A lower bound on it can be easily derived from known results.

Proposition 6. $f_{r}(n) \geq\left\lceil\frac{n}{r-1}\right\rceil$.

Proof. Any $r$-coloring of the blocks of an $\operatorname{STS}(n)$ defines an $r$-coloring of the edges of $K_{n}$, by coloring the three pairs defined by a block with the color of the block. In this coloring there is a monochromatic, say red connected component $C$ with at least $\left\lceil\frac{n}{r-1}\right\rceil$ vertices, proved first in [4], a more accessible account is the survey [5]. The blocks covering the red edges of $C$ obviously span a red connected component on $C$.

The lower bound of Proposition [6 is trivially sharp for $r=2$ but also for certain other values of $r$, starting with $r=4,8,10,14, \ldots$

Proposition 7. $f_{r}(n)=\frac{n}{r-1}$ for infinitely many $n$ if $r-1$ is in the form $3^{m}, p^{m}, q^{2 m}$ where $m \geq 1, p, q$ are primes, $p \equiv 1(\bmod 6), q \equiv-1(\bmod 6)$.

Proof. $\quad f_{r}(n) \geq \frac{n}{r-1}$ follows from Proposition 6. Suppose $r-1$ is a prime power in the form $3^{m}, p^{m}, q^{2 m}$ where $m \geq 1, p, q$ are primes, $p \equiv 1(\bmod 6), q \equiv-1(\bmod 6)$. This implies that $r-1 \equiv 1(\bmod 6)$ or $r-1 \equiv 3(\bmod 6)$. Then there exists an affine plane $P$ of order $r-1$ and we can define an $\operatorname{STS}\left((r-1)^{2}\right)$ by substituting each line of $P$ by a copy of an $\operatorname{STS}(r-1)$. Then the blocks of $\operatorname{STS}\left((r-1)^{2}\right)$ can 
be naturally colored with $r$ colors according to the $r$ parallel classes of $P$. In this coloring every component has size $r-1=\frac{(r-1)^{2}}{r-1}$, providing an example with equality. To get infinitely many, we can apply the well-known direct product construction (see [1]) of $\operatorname{STS}\left(n_{1} n_{2}\right)$ from $\operatorname{STS}\left(n_{1}\right), \operatorname{STS}\left(n_{2}\right)$. Assume we already know that for some $t \geq 0$ the blocks of $\operatorname{STS}\left(3^{t}(r-1)^{2}\right)$ can be $r$-colored so that each color class has $r-1$ nontrivial components (of size $3^{t}(r-1)$ ) and consider the $\operatorname{STS}\left(3^{t+1}(r-1)^{2}\right)$ defined as $\operatorname{STS}\left(3^{t}(r-1)^{2}\right) \times T$ where $T$ is a single block on three points. Then each component $C$ in each color class of $\operatorname{STS}\left(3^{t}(r-1)^{2}\right)$ defines a component $C \times T$ in $\operatorname{STS}\left(3^{t+1}(r-1)^{2}\right)$ whose blocks in $C \times T$ can be colored with the same color. This defines a natural $r$-coloring of the blocks of $\operatorname{STS}\left(3^{t+1}(r-1)^{2}\right)$, preserving the property that each color class has $r-1$ nontrivial components.

Our problem to determine $f(n)$ led to find $G(n)$, the size of the largest cross-free set present in some $\operatorname{STS}(n)$. It seems natural and interesting to find or estimate the size $g(n)$ of the largest cross-free set present in any $\operatorname{STS}(n)$. Obviously,

$$
G(n) \geq g(n) \geq \frac{\alpha(n)}{3}
$$

where $\alpha(n)$ is the largest independent set present in any $\operatorname{STS}(n)$. For the most recent result and history on $\alpha(n)$ see [3].

Problem 8. Is $g(n)$ significantly smaller than $G(n)$ ?

\section{Proof of Theorems 1, 4}

We prove first that $f(6 k+3) \geq 4 k+3, f(6 k+1) \geq 4 k+2$.

Suppose that the blocks of an $\operatorname{STS}(V, \mathcal{B})$ with $|V|=n$ are 3-colored and consider the three components $C_{1}, C_{2}, C_{3}$ in colors $1,2,3$ containing a point $v \in V$. There are some cases according to the number of $C_{i} \mathrm{~s}$ with points covered only by $C_{i}$, we call such points as "private parts" of $C_{i}$.

Case 1. No $C_{i}$ has private part. In this case the sets $C_{i}$ doubly cover $V \backslash\{v\}$ and $v$ is triply covered. This implies easily that $f(6 k+3) \geq 4 k+3$ and also $f(6 k+1) \geq 4 k+2$, unless if the $C_{i}$ s intersect in one point and all the three doubly covered sets have size $2 k$. However, in this case we can have $3 k$ blocks covering $v$ and any other block must cover a pair of $\left(C_{i} \cap C_{j}\right) \backslash\{v\}$. Thus altogether we have at most $3 k+3\left(\begin{array}{c}2 k \\ 2\end{array}\right)<\frac{\left(\begin{array}{c}6 k+1 \\ 2\end{array}\right)}{3}$ blocks in $\operatorname{STS}(6 k+1)$ and that is a contradiction.

Case 2. Only $C_{1}$ has a private part. Now there is no point $w \in V$ that belongs to $\left(C_{2} \cap C_{3}\right) \backslash C_{1}$, otherwise no block can cover $w x$ where $x$ is from the private part of $C_{1}$. Thus in this case $C_{1}$ covers $V$. 
Case 3. Two $C_{i} \mathrm{~s}$, say $C_{1}, C_{2}$ have private parts. Now $\left(C_{1} \cap C_{3}\right) \backslash C_{2}$ and $\left(C_{1} \cap\right.$ $\left.C_{2}\right) \backslash C_{3}$ are both empty and any pair of points $x, y$ from the private parts of $C_{1}, C_{2}$, respectively, must be in a block colored with color 3 . Thus the union of the private parts of $C_{1}, C_{2}$ is part of a component $C$ of color 3 . We can now apply the argument of Case 1 to the components $C, C_{1}, C_{2}$.

Case 4. All $C_{i}$ s have private parts. Now sets covered by precisely two of $C_{1}, C_{2}, C_{3}$ must be empty and the private parts $X_{i} \subset C_{i}$ together with $X_{4}=C_{1} \cap C_{2} \cap C_{3}$, partition $V$. Pairs of points $x \in X_{1}, y \in X_{2}$ must be in a block of color 3 , pairs of points $x \in X_{1}, y \in X_{3}$ must be in a block of color 2, pairs of points $x \in X_{2}, y \in X_{3}$ must be in a block of color 1 , thus the union of any two $X_{i}$ s is covered by (in fact equal to) a monochromatic component. Observe that every block of our $(V, \mathcal{B})$ must contain a pair from some of the $X_{i}$ s, thus

$$
s=\sum_{i=1}^{4}\left(\begin{array}{c}
\left|X_{i}\right| \\
2
\end{array}\right) \geq \frac{\left(\begin{array}{c}
n \\
2
\end{array}\right)}{3} .
$$

First let $n=6 k+3$, assume w.l.o.g that

$$
\left|X_{1}\right| \leq\left|X_{2}\right| \leq\left|X_{3}\right| \leq\left|X_{4}\right|
$$

If $\left|X_{1}\right| \geq 3 k+1+t$ for some positive integer $t$ then let $X_{j}$ be the largest among $X_{2}, X_{3}, X_{4}$. Then

$$
\left|X_{1}\right|+\left|X_{j}\right| \geq 3 k+1+t+\frac{3 k-t+2}{3} \geq 4 k+3
$$

proving what we need. However, if $\left|X_{1}\right| \leq 3 k+1$ then the maximum of $s$ (under the condition that each component has size at most $2 k+2)$ is obtained when $\left|X_{1}\right|=$ $3 k+1,\left|X_{2}\right|=\left|X_{3}\right|=k+1,\left|X_{4}\right|=k$. But this contradicts to (1). Similar argument works if $n=6 k+1$, then

$$
\left|X_{1}\right|=3 k+1,\left|X_{2}\right|=\left|X_{3}\right|=\left|X_{4}\right|=k
$$

gives the largest $s$ and the contradiction.

This finishes the proof of the two inequalities of Theorem 1. It is left to prove that $f(6 k+3)=4 k+3$ if $k$ is divisible by 3 , i.e. to prove Theorem 4 . In fact we need to prove only that $G(n) \geq 6 k$, however $G(n)<6 k+1$ follows easily: a partition of $V$ for a $\operatorname{STS}(18 k+3)$ into three sets of size $6 k+1$ cannot be cross-free since then there are at most $t=3\left(\begin{array}{c}6 k+1 \\ 2\end{array}\right)$ blocks and $t$ is less than the number of blocks required in an $\operatorname{STS}(18 k+3)$.

We construct an STS $(18 k+3)$ with a cross-free set of size $6 k$ as follows. Let $H_{k}$ be the graph with $6 k$ vertices and $4 k$ edges, having $2 k$ components, $k$ of them a $P_{4}$, 
a path on four vertices, and $k$ of them a single edge. We call the middle of $H_{k}$ the union of the middle edges of the $P_{4}$ components in $H_{k}$. A near factor of a graph with $2 m$ (or $2 m-1$ ) vertices means $m-1$ pairwise disjoint edges.

Lemma 9. Let $T$ be the graph containing $k$ vertex disjoint edges on $6 k$ vertices. Then the edge set of $G_{k}=K_{6 k} \backslash T$ can be partitioned into $2 k$ factors $F_{1}, \ldots, F_{2 k}$ and $4 k$ near factors $E_{1}, \ldots, E_{4 k}$ in such a way that the pairs uncovered by the near factors form a graph isomorphic to $H_{k}$ and in the isomorphism the middle of $H_{k}$ corresponds to the pairs of $T$.

Based on the lemma, we define an STS $(18 k+3)$ with a cross-free set of size $6 k$. Take three disjoint copies of $H_{k}$ on vertex sets $X_{0}, X_{1}, X_{2}$ and define $\mathcal{T}$ as a partial triple system $\mathrm{PTS}(18 k)$ on $\cup_{i=0}^{2} X_{i}$ as follows. Partition each $X_{i}$ into $k P_{4}$ paths $a_{6 j+1}^{i}, a_{6 j+2}^{i}, a_{6 j+3}^{i}, a_{6 j+4}^{i}$ and $k$ edges $a_{6 j+5}^{i}, a_{6 j+6}^{i}$ for $j=0, \ldots k-1$. This way each $X_{i}$ spans a copy of $H_{k}$.

Now Lemma 9 can be applied with vertex set $X_{0}$ to obtain $2 k$ factors and $4 k$ near factors with the required properties (with respect to the copy of $H_{k} \subset X_{0}$ ). We can extend these factors and near-factors to blocks of $\mathcal{T}$, using vertices of $X_{1}$ as follows. Let $a_{6 j+4}^{1}$ define blocks with the pairs of the near factor $E_{4 j+1}$ with uncovered pair $a_{6 j+2}^{0}, a_{6 j+3}^{0}, j=0, \ldots, k-1$. Then $a_{6 j+1}^{1}$ defines blocks with the pairs of the near factor $E_{4 j+2}$ with uncovered pair $a_{6 j+5}^{0}, a_{6 j+6}^{0}, j=0, \ldots, k-1$; similarly $a_{6 j+6}^{1}$ defines blocks with the pairs of the near factor $E_{4 j+3}$ with uncovered pair $a_{6 j+1}^{0}, a_{6 j+2}^{0}$, $j=0, \ldots, k-1$; and $a_{6 j+5}^{1}$ defines blocks with the pairs of the near factor $E_{4 j+4}$ with uncovered pair $a_{6 j+3}^{0}, a_{6 j+4}^{0}, j=0, \ldots, k-1$. Finally, $a_{6 j+2}^{1}, a_{6 j+3}^{1}$ define blocks with the pairs of the factors $F_{2 j+1}, F_{2 j+2}, j=0, \ldots, k-1$.

The construction of the previous paragraph can be repeated cyclically, defining blocks with one vertex in $X_{2}$ and two in $X_{1}$, and a third time defining blocks with one vertex in $X_{0}$ and two in $X_{2}$. By Lemma 9, the partial STS $\mathcal{T}$ defined this way covers all pairs of $X_{0} \cup X_{1} \cup X_{2}$ except a 3-regular graph $U$ of with the following edges: $a_{6 j+2}^{i}, a_{6 j+3}^{i}$ for $i=0,1,2$ and $j=0, \ldots, k-1$ (formed by the middle of the three copies of $H_{k}$ ) and the $3 \times 8 k$ edges between the pairs $X_{i}, X_{j}$ that belong to the uncovered pairs of the $3 \times 4 k$ near factors. It can be easily seen that the graph $U$ can be factored into three 1 -factors. In fact, these factors are

$$
\begin{aligned}
& a_{6 j+2}^{i}, a_{6 j+3}^{i}, a_{6 j+1}^{i}, a_{6 j+6}^{i-1}, a_{6 j+5}^{i}, a_{6 j+4}^{i-1}, \\
& a_{6 j+4}^{i}, a_{6 j+2}^{i-1}, a_{6 j+5}^{i}, a_{6 j+3}^{i-1}, a_{6 j+6}^{i}, a_{6 j+1}^{i-1}, \\
& a_{6 j+1}^{i}, a_{6 j+5}^{i-1}, a_{6 j+4}^{i}, a_{6 j+3}^{i-1}, a_{6 j+6}^{i}, a_{6 j+2}^{i-1},
\end{aligned}
$$

where $i=0,1,2$ and $j=0, \ldots k-1$ with arithmetic on $i, j$-s are modulo 3,6 , respectively. 
Finally, $\mathcal{T}$ is extended to an $\operatorname{STS}(18 k+3)$ by extending each factor of $U$ to a block with one of three new points $A, B, C$ which also forms the last block. This finishes the proof of Theorem 4 and with it Theorem 1 .

Proof of Lemma 9. The required partition is constructed from the standard factorization of $K_{6 k}$ on vertex set $\{1,2, \ldots, 6 k-1\} \cup \infty$ where (for $i=1,2, \ldots, 6 k-1$ ) factor $F_{i}$ contains $(i, \infty)$ and $\{(i-j, i+j): 1 \leq j \leq 3 k-1\}$ with $\bmod 6 k-1$ arithmetic.

We shall keep $2 k-1$ of the factors $F_{i}$ and define the near factors $E_{1}, \ldots, E_{4 k}$ by deleting one edge from each of the other $4 k$ factors so that the deleted edges form a graph isomorphic to $H_{k}$. The factor formed by the middle of $H_{k}$ is left uncovered and all other edges of $H_{k}$ form a new factor $F^{*}$ which is added as the $2 k$-th factor in the partition. To define the construction, it is enough to specify the set of $4 k$ pairs (all from different $F_{i}$ ) which form a graph $Z_{k}$ isomorphic to $H_{k}$. The construction is the simplest for $k \equiv 1 \quad(\bmod 2)$ so we describe that first.

Suppose that $k \equiv 1 \quad(\bmod 2)$ and set $W=\{(1,3),(2,4),(3,5),(5, \infty)\}$. Moreover, for $m \in\{6,12, \ldots, 6(k-2)\}$ let $L_{m}=A_{m} \cup B_{m}$ be the following set of eight pairs on twelve consecutive numbers:

$$
\begin{gathered}
A_{m}=\{(m, m+2),(m+2, m+4),(m+4, m+6),(m+1, m+3)\}, \\
B_{m}=\{(m+5, m+7),(m+7, m+9),(m+9, m+11),(m+8, m+10)\} .
\end{gathered}
$$

It is immediate to check that $W, A_{m}, B_{m}$ are all define (6-vertex) graphs with a $P_{4}$ component and a $K_{2}$ component. Thus the graph $Z_{k}$ defined by $W$ (for $k=1$ ) and by $W \cup_{m=6}^{6(k-2)} L_{m}$ (for odd $k>1$ ) is isomorphic to $H_{k}$. Moreover, since all edges (apart from $(5, \infty))$ of $Z_{k}$ are in the form $(j, j+2)$ and $j \neq 4$, each edge of $Z_{k}$ belongs to different $F_{i}$.

The case $k \equiv 0 \quad(\bmod 2)$ is slightly more involved, we use another type of components $C_{m}, D_{m}$ (beside $W$ ) to define $Z_{k}$.

$$
\begin{gathered}
C_{m}=\{(m, m+1),(m, m+2),(m+2, m+4),(m+3, m+5)\}, \\
D_{m}=\{(m, m+2),(m+1, m+2),(m+1, m+3),(m+4, m+5)\} .
\end{gathered}
$$

For $k=2$ we use $W$ followed by $C_{6}$ to define $Z_{2}$. For $k>2$ start with $W$, then $\frac{k}{2}$ copies of $C_{m}(m=6,12, \ldots, 3 k)$ then $\frac{k-2}{2}$ copies of $D_{m}(m=3 k+6, \ldots, 6(k-1))$. To check here that each edge of $Z_{k}$ belongs to different $F_{i}$, note that "jumping pairs" $(j, j+2)$ are obviously from different $F_{i}$ (from $F_{j+1}$ ). The same is true for the "consecutive pairs" $(j, j+1)$. To check consecutive pairs against jumping pairs, notice that for $m=6,12, \ldots, 3 k$ the pair $(m, m+1)$ of $C_{m}$ belongs to $F_{3 k+m}$, a starting point of the $D$-block opposite to $C_{m}$ thus it is not skipped by any jumping pair. Similarly, for 
$m=3 k+6, \ldots, 6(k-1)$, the pairs $(m+1, m+2)$ and $(m+4, m+5)$ in $D_{m}$ belong to $F_{m+2-3 k}$ and $F_{m+5-3 k}$, respectively, and they are not skipped in their opposite $C$-blocks.

Acknowledgement. Thanks for the remarks of the referees, especially for calling my attention to [2], showing the connection of 3-bichromatic Steiner triple systems and the ones with large cross-free sets.

\section{References}

[1] C. J. Colbourn, A. Rosa, Triple systems, Calendron Press, Oxford, 1999.

[2] C. J. Colbourn, J. H. Dinitz, A. Rosa, Bicoloring Steiner triple systems, Electronic Journal of Combinatorics, 6 (1999) R25.

[3] A. Eustis, J. Verstraëte, On the independence number of Steiner systems, Combinatorics, Probability and Computing to appear

[4] A. Gyárfás, Partition coverings and blocking sets in hypergraphs (in Hungarian) Communications of the Computer and Automation Institute of the Hungarian Academy of Sciences 71 (1977) 62 pp.

[5] A. Gyárfás, Large monochromatic components in edge colorings of graphs: A survey. Book chapter in Ramsey Theory, yesterday, today and tomorrow, Progress in mathematics 285, Ed: A. Soifer Birkhauser, 2011 pp. 77-96. 